\title{
Experiência Pedagógica em Patologia na Faculdade de Medicina da UFC
}

\section{Educational Experience in Pathology at the UFC Medical School}

\author{
Daniela Cristina Sensato Monteiro \\ José Udevanier Rebouças da Silva Júnior ${ }^{I}$ \\ Loraine Correia Vieira Matos ${ }^{I}$ \\ Margarida Maria de Lima Pompeu ${ }^{I}$ \\ Conceição Aparecida Dornelas ${ }^{I}$
}

PALAVRAS-CHAVE

- Patologia;

- Aprendizagem;

- Motivação;

- Educação Médica.

\section{KEYWORDS}

- Pathology;

- Learning;

- Motivation;

- Medical Education.

\section{RESUMO}

Objetivo: Relatar a experiência da aplicação de material didático composto por catálogo com imagens macroscópicas e microscópicas, disponível em meio impresso e digital, acompanhado de coleções de lâminas, instrumento criado por alunos do mestrado e iniciação científica, focando a autonomia do aprendizado em Patologia Humana para os alunos da graduação. Métodos: Foi elaborado um questionário para ser aplicado aos alunos, para avaliação de resultados alcançados pelo modelo criado, que será oferecido aos estudantes de diversos cursos que possuem a disciplina de Patologia Humana, oferecida na Faculdade de Medicina da Universidade Federal do Ceará (UFC). O catálogo relata a história e evolução da doença de uma paciente com tuberculose sistêmica, suas manifestações clínicas, exames físicos e laboratoriais, além de achados de necropsia, com diferentes aspectos da resposta inflamatória. Resultados: Os alunos que viveram esta experiência se manifestaram a favor da implantação da nova ferramenta como material didático da disciplina e sua manutenção nos laboratórios. Conclusão: $A$ inovação nas aulas é necessária, e este modelo surge como um material complementar para a melhoria e motivação das aulas práticas no tema proposto.

\begin{abstract}
Objective: To reporting on the application of new teaching material composed of a catalog with macroscopic and microscopic images, available in print and digital media, accompanied by slides of collections. This instrument was created by Master's and Scientific Initiation students, focusing on the undergraduate students' autonomy in learning Human Pathology. Methods: A questionnaire was developed to be administered to students to assess the results achieved by the new model, which will be available to students whose courses require attending Human Pathology classes at the Federal University of Ceará (UFC) Medical School. The catalog describes the history and evolution of the disease in a patient with systemic tuberculosis, its clinical manifestations, physical and laboratorial tests and autopsy findings, with different aspects of the inflammatory response. Results: The students who assessed this model were in favor of its implementation as teaching material for Human Pathology classes and its maintenance in laboratories. Conclusion: Innovation in classes is necessary and this model emerges as supplementary material for the improvement and motivation of practical classes on the proposed theme.
\end{abstract}

Recebido em: 08/01/2014

Aprovado em: 29/05/2015 


\section{INTRODUÇÃO}

Em diversos países, desde a segunda metade do século XX, a formação de profissionais da saúde vem, cada vez mais, sendo motivo de frequentes discussões ${ }^{1}$. A formação tradicional em saúde adota um ensino organizado em disciplinas, centrado no professor, com atividades práticas no cenário excessivamente hospitalar, que é marcado pela unidirecionalidade na relação professor-estudante e pela fragmentação do corpo e da saúde das pessoas, baseada no modelo elaborado por Flexner² em 1910.

Atualmente, as escolas mais inovadoras utilizam o ensino e o aprendizado centrado no aluno, no qual a busca do aprendizado é feita pelo próprio aluno. $\mathrm{O}$ autoaprendizado pode ser facilitado pela metodologia de ensino em pequenos grupos e assistido por computadores, favorecendo o processo de autoavaliação ${ }^{3}$.

A aprendizagem pode ser definida como o resultado da estimulação do ambiente sobre o indivíduo já maduro, que se expressa diante de uma situação-problema, sobre a forma de mudança de comportamento em função da experiência. A aprendizagem não pode ser vista como apenas um processo de memorização, que utiliza apenas o conjunto das funções mentais ou somente os elementos físicos ou emocionais, pois todos estes aspectos são necessários ${ }^{4}$. No processo de aprendizagem, não só livros-textos são importantes, é preciso utilizar outras ferramentas que despertem a motivação dos alunos, diversificando as ferramentas de ensino.

A educação tem passado por reformas nos últimos anos, na tentativa de garantir uma aprendizagem mais eficiente e interessante, aperfeiçoando tecnologias para uma relação pedagógica progressiva, motivadora e interativa. A educação deve caminhar com a modernização por meio dos novos recursos da informatização ${ }^{5}$.

A utilização de ferramentas inovadoras nos laboratórios de microscopia em associação com sistemas digitais (computadores, mídias digitais) pode facilitar o aprendizado universitário. Com o intuito de melhorar a qualidade de ensino nos laboratórios de microscopia, várias universidades têm buscado nas Tecnologias de Informação e de Comunicação soluções que tragam aos alunos um melhor aproveitamento das aulas ${ }^{6,7}$. A utilização de recursos multimídia em aulas de microscopia tem o benefício de apresentar imagens microscópicas no foco e com alta qualidade, o que permite uma redução no tempo laboratorial, pois contribui para a discussão em grupo de estruturas histológicas apresentadas pelo professor, geralmente difícil de promover quando os alunos estudam cada um em seu microscópio ${ }^{7,8}$.

Para colaborar com a inovação didática, educadores devem considerar a combinação de outras estratégias de ensino para a construção do conhecimento de seus alunos. Portanto, a utilização de materiais pedagógicos mais interativos contribuiria para melhor rendimento de aprendizagem.

Sempre que possível, as mudanças nas práticas pedagógicas em sala de aula devem acompanhar o avanço tecnológico, visto que a tecnologia está envolvida no dia a dia dos alunos e que o processo de ensino-aprendizagem tem que caminhar de acordo com a realidade do aluno no seu cotidiano 9 . Não se deve prender a práticas rotineiras, estereotipadas, muitas vezes alicerçadas em ideias simplificadas, quase clichês, que perderam o potencial para análise crítica da realidade e do enfrentamento dos problemas educacionais ${ }^{10}$.

O Laboratório de Investigação Diagnóstica do Departamento de Patologia e Medicina Legal (DPML) da Universidade Federal do Ceará (UFC) busca dar suporte à metodologia de aprendizagem baseada em problemas (ABP) desenvolvida no terceiro semestre do curso de Medicina e trabalhar as competências dos módulos dos semestres clínicos. O DPML busca desenvolver estações interativas que contenham peças (modelos, lâminas, espécimes clínicos e materiais instrucionais digitais), abordando os mecanismos fisiopatológicos e de investigação diagnóstica das doenças estudadas nas disciplinas de Patologia dos cursos de Medicina, Farmácia, Odontologia e Enfermagem.

Sabemos que a introdução de uma nova ferramenta voltada para a autoaprendizagem é um desafio, necessitando da aceitação dos alunos e professores, mas pode contribuir para a inovação do ensino no laboratório. Assim, este trabalho relata, por meio de um questionário, a opinião dos alunos sobre a construção de um material didático composto de um catálogo de imagens, acompanhado por caixas de lâminas e mídia digital (DVD com imagens de microscopia).

O tema escolhido para este material de autoaprendizagem, a ser utilizado na disciplina de Patologia Humana, foi a tuberculose, pela importante característica do processo básico patológico, que deve ser bem compreendido, e pela alta incidência em nosso país. Assim, foi intitulado: "Fisiopatologia da tuberculose - abordagem para a autonomia do aprendizado: catálogo de imagens acompanhando coleção de lâminas e DVD".

A tuberculose é conhecida por ser uma calamidade neglicenciada $^{11}$, sendo considerada um problema de saúde pública. A pobreza e a desigualdade social contribuem para a alta incidência desta doença. Atualmente, ela representa a segunda causa de morte por doença infecciosa em todo o mundo, depois do vírus da imunodeficiência humana (HIV $)^{12}$.

O Brasil está entre os 22 países que concentram $82 \%$ dos casos de tuberculose no mundo ${ }^{13}$. Estima-se que ocorram anu- 
almente 129 mil casos novos de tuberculose no Brasil, dos quais cerca de 40 mil não são notificados, por isso trata-se de uma prioridade nos programas nacionais de saúde ${ }^{14}$. Estima-se que, entre 2002 e 2020, mais de 150 milhões de pessoas ficarão doentes, e 36 milhões irão morrer, principalmente nos países em desenvolvimento ${ }^{15}$. Em 2013, o Brasil diagnosticou 71.123 casos novos de tuberculose, perfazendo um coeficiente de incidência de 35,4/100.000 habitantes ${ }^{16}$.

Pensando nesse cenário e na importância de seu aprendizado no contexto local e nacional, o material foi criado para oferecer ao estudante não apenas um acervo de lâminas, mas também um catálogo, uma rica fonte de estudo sob a forma de uma estação interativa, por intermédio da qual poderá revisar o conteúdo de acordo com a necessidade para o processo de aprendizagem.

\section{OBJETIVO}

Relatar a avaliação dos alunos sobre a experiência de uso de um novo instrumento pedagógico (catálogo, caixa de lâminas, mídia digital), focando a autonomia do aprendizado em Patologia Humana dos alunos da graduação dos cursos de Enfermagem, Farmácia, Odontologia e Medicina da UFC.

\section{METODOLOGIA}

\section{Avaliação do material de autoaprendizagem pelos alunos}

Elaborou-se um questionário para ser aplicado aos alunos do terceiro semestre do curso de Medicina da UFC, para avaliação das ferramentas de autoaprendizagem desenvolvidas sobre a fisiopatologia da tuberculose.

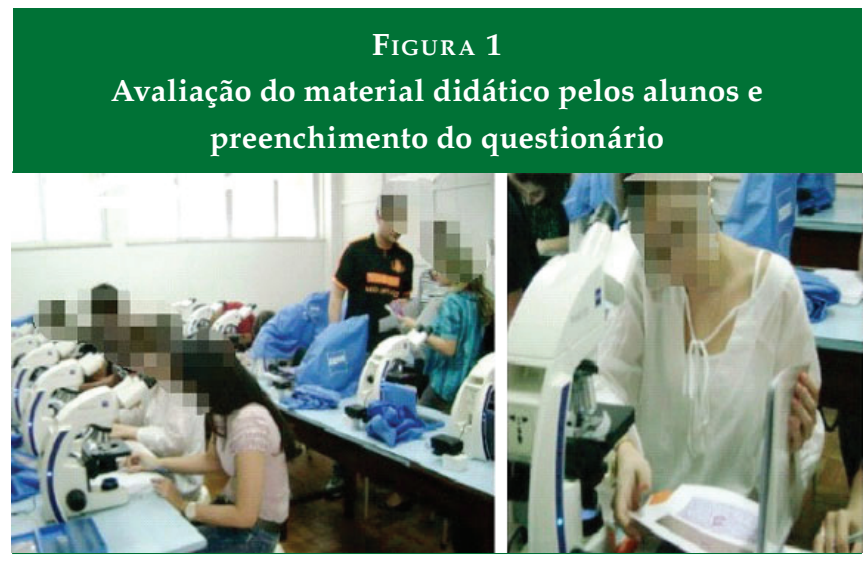

Os alunos foram convidados a participar e recebidos no laboratório de microscopia com o material (lâminas e catálogo - Figura 1) já disposto e organizado sobre as bancadas.
Detalhou-se a ideia do que seria o novo material, como foi a sua construção e seu objetivo. Após minuciosa observação por parte dos alunos participantes, foi solicitado que respondessem a um questionário.

O questionário era composto por oito questões simples, que avaliavam a receptividade do material pelos alunos, a qualidade, a importância de inovações e novas tecnologias para o ensino e o hábito de estudo desses alunos - por exemplo, se utilizavam apenas livros recomendados pelo professor em aula ou se buscavam livros auxiliares. Os alunos envolvidos no projeto foram responsáveis pela aplicação do questionário.

\section{Construção do material de autoaprendizagem}

A construção do material de autoaprendizagem envolveu uma aluna do mestrado em Patologia, dois alunos de iniciação científica do curso de Medicina e duas professoras, orientadora e co-orientadora, todos da UFC.

O material didático proposto é composto de um catálogo sobre a fisiopatologia da tuberculose, com fotografias macroscópicas e microscópicas das amostras e instruções detalhadas para o correto manuseio do microscópio. Também foram construídas caixas de lâminas de cada órgão e mídia digital (DVD com imagem das lâminas).

\section{Imagens macroscópicas}

Foram coletadas imagens no Departamento de Medicina Legal da UFC de peças de uma paciente com tuberculose sistêmica.

As peças utilizadas na construção do catálogo foram lavadas e colocadas sobre a bancada para serem fotografadas (Figura 2). Foram fotografados pulmão, fígado, rim, baço, omento e intestino delgado. Para a captação das imagens, utilizou-se uma câmera Sony, modelo Alfa 230.

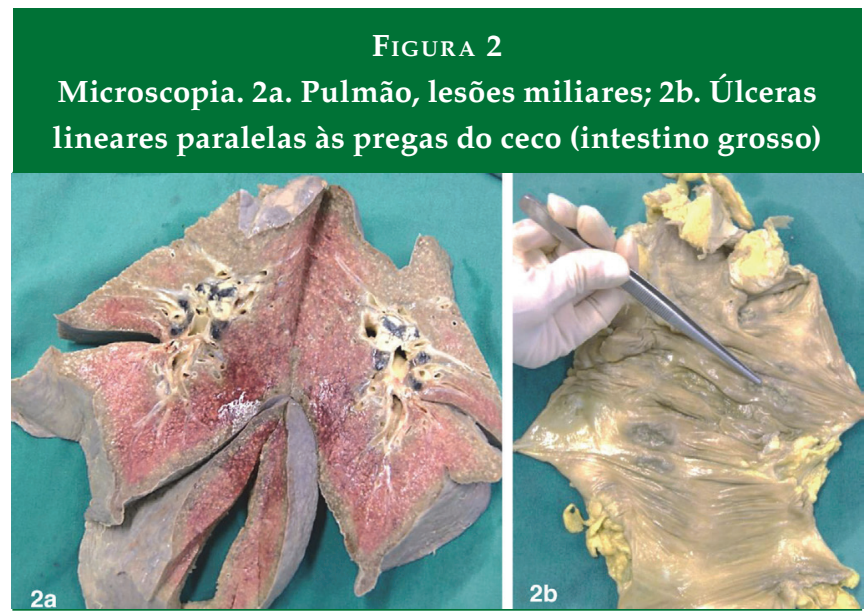




\section{Imagens microscópicas}

Para a confecção das lâminas de microscopia, removeram-se fragmentos de cerca de $2 \mathrm{~cm} \times 2 \mathrm{~cm}$ dos órgãos de interesse. Em seguida, foram transferidos para cassetes e posteriormente incluídos em parafinas em histotécnico. Os blocos foram cortados em micrótomo com a espessura de $5 \mu \mathrm{m}$ para a confecção das lâminas, coradas por hematoxilina/eosina.

De cada fragmento foram confeccionadas de 80 a 100 lâminas de cada órgão, totalizando 12 caixas de lâminas. Após serem confeccionadas, as lâminas foram fotografadas para a construção do catálogo de autoaprendizagem (Figura 3). Foram feitas lâminas de pulmão, fígado, rim, baço, omento, intestino delgado, tireoide e foco de Ghon.

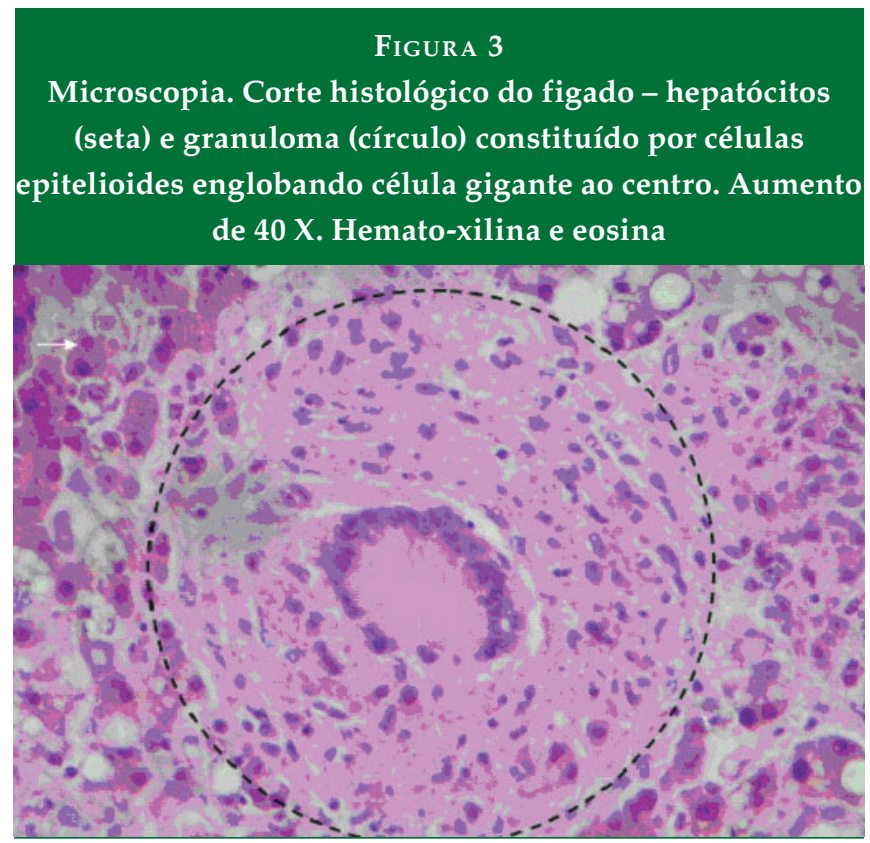

\section{Construção do catálogo}

A capa/arte do catálogo foi gentilmente criada pelo professor da Faculdade de Medicina da UFC, Prof. Dr. Francisco Hélio Rôla, médico e artista plástico. Em virtude de sua boa integração e relação com os alunos, sempre envolvido em projetos de artes plásticas da universidade, foi convidado a fazer a capa/ arte deste material (Figura 4).

Para a produção de textos, utilizou-se o programa Microsoft Publisher. Após o sumário, foram inseridas informações detalhadas sobre a forma correta de utilização do microscópio. Como ilustração, usou-se uma foto do microscópio utilizado nos laboratórios da UFC, com as partes de cada um dos componentes do microscópio detalhadas. Em seguida, uma breve introdução sobre a doença da tuberculose e granulomas com as referências utilizadas. Foram distribuídas, então, as imagens macroscópicas e microscópicas de cada órgão, com observações e marcações quando necessário. No final, foi colocado o relato de caso da paciente e o laudo anatomopatológico da necropsia.
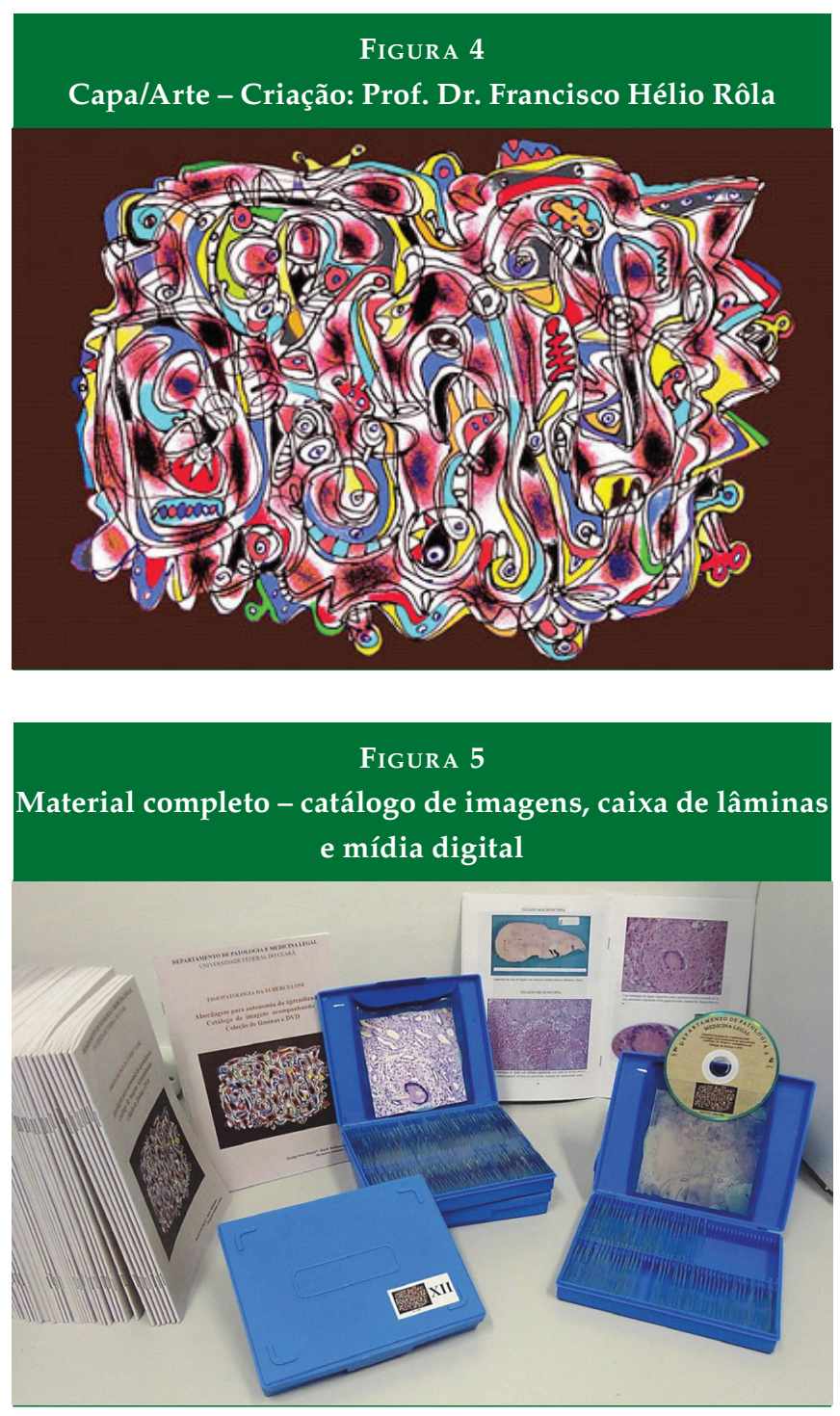

Foram gravadas as mídias digitais (DVDs), com todas as imagens macroscópicas e microscópicas contidas no catálogo. Foram colocadas dentro de cada caixa de lâmina, para serem utilizadas também como ferramenta pelo professor durante a aula e posteriormente pelos alunos para estudo individual.

Os 80 catálogos foram gentilmente impressos pela Gráfica da UFC e, juntamente com 12 caixas de lâminas com cortes de diversos órgãos, contendo mídia digital de imagens em cada caixa, foram colocados à disposição dos alunos e professores na Sala de Microscopia III da UFC (Figura 5). 


\section{RESULTADOS}

Os alunos responderam voluntariamente a 86 questionários durante a apresentação do material. Quando foram questionados sobre a importância de um catálogo com diversas fotos de macroscopia e microscopia no seu aprendizado sobre a fisiopatologia e clínica da tuberculose, 88,3\% (76) consideraram muito importante, e 11,7\%(10), importante.

A respeito da autonomia que o catálogo disponibiliza no processo de aprendizagem, 95,3\% (82) disseram sim, enquanto apenas 4,7\% (4) apontaram como indiferente.

Quanto à orientação sobre o correto uso do microscópio contida no catálogo, 89,5\% (77) disseram que foram orientados, 1,2\% (1) relatou que não houve orientação e 9,3\% (8) foram indiferentes.

Dos alunos participantes, 51,2\% (44) disseram ter o hábito de estudar apenas por livros recomendados pelo professor e $48,8 \%$ (42) gostam de aprofundar o aprendizado com outros livros.

Todos os alunos - (100\%) 86 - responderam que têm interesse por materiais de autoaprendizagem; 74,4\% (64) disseram que o Departamento estimula a autonomia do aluno no processo de aprendizagem, mas 25,6\% (22) responderam que não há estímulo à autonomia. Perguntados se o Departamento disponibiliza materiais que possibilitem essa autonomia, 41,8\% (36) disseram que sim, enquanto 58,1\% (50) afirmaram que não.

Dos 86 questionários preenchidos que avaliaram o material disponibilizado, 90,7\% (78) consideraram o material ótimo, e $9,3 \%$ (8), bom.

\section{CONCLUSÃO}

Na avaliação do questionário, 100\% (86) dos alunos se manifestaram a favor da implantação da nova ferramenta como material didático da disciplina e também da manutenção desse instrumento nos laboratórios, assim como de sua extensão a outros temas e até a outras disciplinas em que se utiliza microscopia na UFC.

A inovação do material didático nas aulas é necessária e contribui para a melhoria e motivação das aulas práticas e a compreensão dos temas abordados. Após a aula, os alunos continuam com esse material (caixas de lâminas e catálogos) a sua disposição no laboratório para o processo de autoaprendizagem, podendo revisar e compreender melhor o conteúdo quantas vezes for necessário. O material foi bem aceito pelos alunos do DPML e já se encontra à disposição de alunos e professores.

Embora a maioria dos alunos considere que o Departamento estimula a autoaprendizagem, este não disponibiliza tantos materiais com esse fim. Assim, é necessário o desenvolvimento de novas ferramentas nesse campo pelo Departamento.

\section{REFERÊNCIAS}

1. Pereira OP, Almeida TMC. A formação médica segundo uma pedagogia de resistência. Interface- Comunic., Saúde, Educ. 2005; 19(16):69-79.

2. Flexner A. Medical education in the United States and Canada. San Francisco: Carnegie Foundation for Higher Education; 1910.

3. Mattos MCI. Ensino Médico: O que sabemos? Interface-Comunic., Saúde, Educ.1997; (1):193-196.

4. José EA, Coelho MT. Problemas de Aprendizagem. São Paulo: Ática; 1999.

5. Fonseca LMM, Goes FSN, Ferecini GM, Leite AM, Mello DF, Scochi CG. Revista Texto Contexto Enfermagem, Florianopolis. 2009; 18(3):549-58.

6. Kumar RK, Freeman B, Velan GM, Permentier PJ. Integrating Histology and Histopathology teaching in Practical Classes Using Virtual Slides. The Anatomical Record. 2006; (289B):128-33.

7. Santa-Rosa JG, Struchiner M. Tecnologia Educacional no Contexto do Ensino de Histologia: Pesquisa e Desenvolvimento de um Ambiente Virtual de Ensino e Aprendizagem. Revista Brasileira de Educação Médica. 2011; 35(2):289-298.

8. Downing SW. A multimídia-based histology laboratory course: elimination of the tradicional microscope laboratory. Medinfo. 1995; 8 (pt 2):1695.

9. Fornaziero CC, Gil CRR. Novas tecnologias aplicadas ao ensino da anatomia humana. Revista Brasileira da Educação Médica. 2003; 27(2):143-146.

10. Basso IS. Significado e sentido do trabalho docente. Cad. CEDES. 1998;19(44).

11. Ruffino-Neto A. Tuberculose: a calamidade negligenciada. Rev Soc Bras Med Trop. 2002; 35 (1):51-8.

12. Prince KA. Epidemiologia clássica e molecular da tuberculose pulmonar em pacientes da região norte de Minas Gerais. Araraquara; 2013. Doutorado [Tese] - UNESP.

13. Farias EJS, Albuquerque IMN, Araújo RA, Soares JSA, Linhares MSC. Análise epidemiológica dos casos de tuberculose notificados no município de Sobral - Ce no período de 2007 a 2011. SANARE. 2013;12(1):33-39.

14. Azedo RCM, Bezerra SS, Fonte CFV, Soares Sobrinho JL. Avaliação comparativa do perfil e tratamento de pacientes nos Programas de Tuberculose do Estado de Pernambuco e do Hospital Universitário Oswaldo Cruz. Boletim Informativo Geum. 2014; 4(1):71-78.

15. World Health Organization, 2011. Disponível em: <http:/ / www.wpro.who.int/sites/stb/.>[Acesso em 29 set. 2013].

16. Brasil. Ministério da Saúde. Secretaria de Vigilância em Saúde. Boletim Epidemiológico. 2014; 45(2). Disponível 
em http://portalsaude.saude.gov.br/images/pdf/2014/ maio/29/BE-2014-45--2--tb.pdf. [Acesso em: 05 out 2014].

\section{CONTRIBUIÇÃO DOS AUTORES}

Daniela Cristina Sensato Monteiro contribuiu para a elaboração do estudo, construção e organização do material didático, aplicação do questionário aos alunos da graduação, interpretação dos dados do questionário e elaboração da versão final e encaminhamento para à publicação. José Udevanier Rebouças da Silva Júnior contribuiu para construção e organização do material didático, aplicação do questionário aos alunos da graduação, interpretação dos dados do questionário. Loraine Correia Vieira Matos contribuiu para montagem do material didático e aplicação do questionário aos alunos da graduação. Margarida Maria de Lima Pompeu contribuiu no desenho inicial do estudo orientando e revisando. Co-orientadora do projeto. Conceição Aparecida Dornelas contribuiu para a elaboração do estudo, construção e organização do material didático, responsável pelas imagens macroscópicas e microscópicas do catalogo, interpretação dos dados do questionário e elaboração da versão final com revisões críticas. Orientadora do projeto.

\section{CONFLITO DE INTERESSES}

Os autores declaram não ter conflito de interesses.

\section{ENDEREÇO PARA CORRESPONDÊNCIA}

Daniela Cristina Sensato Monteiro

Rua Suíça, 196

Residencial Alphaville Eusébio - Eusébio

CEP 61760-903 - CE

E-mail: daniela.monts@gmail.com 